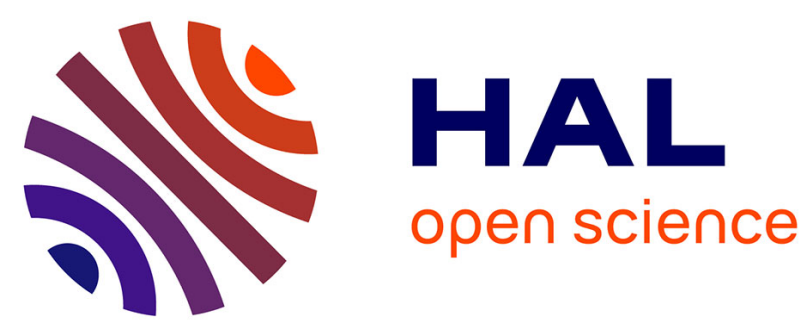

\title{
Novel VCP mutations expand the mutational spectrum of frontotemporal dementia
}

\author{
Dario Saracino, Fabienne Clot, Agnès Camuzat, Vincent Anquetil, Didier \\ Hannequin, Lucie Guyant-Maréchal, Mira Didic, Léna Guillot-Noël, Daisy \\ Rinaldi, Morwena Latouche, et al.
}

\section{To cite this version:}

Dario Saracino, Fabienne Clot, Agnès Camuzat, Vincent Anquetil, Didier Hannequin, et al.. Novel VCP mutations expand the mutational spectrum of frontotemporal dementia. Neurobiology of Aging, 2018, 72, pp.187.e11 - 187.e14. 10.1016/j.neurobiolaging.2018.06.037 . hal-03578014

\section{HAL Id: hal-03578014 https://hal.science/hal-03578014}

Submitted on 16 Feb 2022

HAL is a multi-disciplinary open access archive for the deposit and dissemination of scientific research documents, whether they are published or not. The documents may come from teaching and research institutions in France or abroad, or from public or private research centers.
L'archive ouverte pluridisciplinaire HAL, est destinée au dépôt et à la diffusion de documents scientifiques de niveau recherche, publiés ou non, émanant des établissements d'enseignement et de recherche français ou étrangers, des laboratoires publics ou privés. 


\section{Accepted Manuscript}

Novel VCP mutations expand the mutational spectrum of frontotemporal dementia

Dario Saracino, MD, Fabienne Clot, PhD, Agnès Camuzat, MSc, Vincent Anquetil, $\mathrm{PhD}$, Didier Hannequin, MD, PhD, Lucie Guyant-Maréchal, MD, PhD, Mira Didic, MD, PhD, Léna Guillot-Noël, MSc, Daisy Rinaldi, PhD, Morwena Latouche, PhD, Sylvie

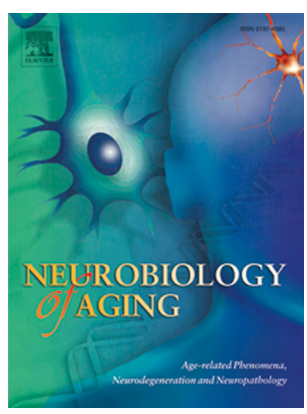
Forlani, PhD, Yassaman Ghassab, BSc, Cinzia Coppola, MD, PhD, Giuseppe Di lorio, $\mathrm{MD}$, Isabelle David, Eric Le Guern, MD, PhD, Alexis Brice, MD, Isabelle Le Ber, MD, $\mathrm{PhD}$

PII: S0197-4580(18)30242-2

DOI: 10.1016/j.neurobiolaging.2018.06.037

Reference: NBA 10309

To appear in: Neurobiology of Aging

Received Date: 30 April 2018

Revised Date: 26 June 2018

Accepted Date: 26 June 2018

Please cite this article as: Saracino, D., Clot, F., Camuzat, A., Anquetil, V., Hannequin, D., GuyantMaréchal, L., Didic, M., Guillot-Noël, L., Rinaldi, D., Latouche, M., Forlani, S., Ghassab, Y., Coppola, C., Di lorio, G., David, I., The French research network on FTD/FTD-ALS, Le Guern, E., Brice, A., Le Ber, I., Novel VCP mutations expand the mutational spectrum of frontotemporal dementia, Neurobiology of Aging (2018), doi: 10.1016/j.neurobiolaging.2018.06.037.

This is a PDF file of an unedited manuscript that has been accepted for publication. As a service to our customers we are providing this early version of the manuscript. The manuscript will undergo copyediting, typesetting, and review of the resulting proof before it is published in its final form. Please note that during the production process errors may be discovered which could affect the content, and all legal disclaimers that apply to the journal pertain. 
Authors. Dario Saracino, $\mathrm{MD}^{1,2,3}$; Fabienne Clot, $\mathrm{PhD}^{4}$; Agnès Camuzat, $\mathrm{MSc}^{1,5}$; Vincent Anquetil, $\mathrm{PhD}^{1}$; Didier Hannequin, $\mathrm{MD}, \mathrm{PhD}^{6}$; Lucie Guyant-Maréchal, $\mathrm{MD}, \mathrm{PhD}^{6}$; Mira Didic, $\mathrm{MD}, \mathrm{PhD}^{7,8}$; Léna GuillotNoël, MSc ${ }^{1}$; Daisy Rinaldi, $\mathrm{PhD}^{1,3}$; Morwena Latouche, $\mathrm{PhD}^{1,}$; Sylvie Forlani, $\mathrm{PhD}^{1}$; Yassaman Ghassab, $\mathrm{BSc}^{1}$; Cinzia Coppola, $\mathrm{MD}, \mathrm{PhD}^{2}$; Giuseppe Di Iorio, $\mathrm{MD}^{2}$; Isabelle David ${ }^{4}$; The French research network on FTD/FTD-ALS*; Eric Le Guern, MD, $\mathrm{PhD}^{1,4}$; Alexis Brice, $\mathrm{MD}^{1,9}$; Isabelle Le Ber, MD, $\mathrm{PhD}^{1,3}$.

Affiliations. ${ }^{1}$ Sorbonne Universités, UPMC Univ Paris 06, Inserm U1127, CNRS UMR 7225, Institut du Cerveau et la Moelle épinière (ICM), AP-HP - Hôpital Pitié-Salpêtrière, Paris, France. ${ }^{2}$ Second Division of Neurology, Department of Medical, Surgical, Neurological, Metabolic and Aging Sciences, University of Campania "Luigi Vanvitelli", Naples, Italy. ${ }^{3}$ Centre de référence des démences rares ou précoces, IM2A, Département de Neurologie, AP-HP - Hôpital Pitié-Salpêtrière, Paris, France. ${ }^{4}$ UF de Neurogénétique Moléculaire et Cellulaire, Département de Génétique, AP-HP - Hôpital Pitié-Salpêtrière, Paris, France. ${ }^{5}$ EPHE, PSL research University, Paris, France. ${ }^{6}$ Department of neurology, CNR-MAJ, Rouen University Hospital, Rouen, France ${ }^{7,8}$ Aix-Marseille Université, INSERM, INS UMR_S 1106, 13005, Marseille, France; ${ }^{8}$ Service de Neurologie et Neuropsychologie, APHM Hôpital Timone Adultes, Marseille, France; ${ }^{9}$ National reference center for neurogenetics, APHP, Hôpital Pitié-Salpêtrière, Paris, France

\section{* The French clinical and genetic research network on FTLD/FTLD-ALS includes:}

Alexis Brice (Hôpital de la Salpêtrière, Paris), Sophie Auriacombe (CHU Pellegrin, Bordeaux), Frédéric Blanc (Hôpitaux Civils, Strasbourg), Philippe Couratier (CHU Limoges), Mira Didic (CHU La Timone, Marseille), Bruno Dubois (Hôpital de la Salpêtrière, Paris), Charles Duyckaerts (Hôpital de la Salpêtrière, Paris), Marie-Odile Habert (Hôpital de la Salpêtrière, Paris), Véronique Golfier (CHU Rennes), Didier Hannequin (CHU Charles Nicolle, Rouen), Lucette Lacomblez (Hôpital de la Salpêtrière, Paris), Isabelle Le Ber (Hôpital de la Salpêtrière, Paris), Richard Levy (Hôpital de la Salpêtrière, Paris), Bernard-François 
Michel (CH Sainte-Marguerite, Marseille), Florence Pasquier (CHU Roger Salengro, Lille), Catherine Thomas-Anterion (CHU Bellevue, Saint-Etienne), Jérémie Pariente (CHU Rangueil, Toulouse), François Sellal (CH Colmar), Martine Vercelletto (CHU Laennec, Nantes).

Corresponding author. Isabelle Le Ber. Address: Institut du cerveau et la moelle (ICM), Pitié-Salpêtrière Hospital, 47-83, boulevard de l'Hôpital, 75651 Paris Cedex 13, France. Phone: 0033157274682 ; Fax: 0033 15727 4795; E-mail: isabelle.leber@upmc.fr

Word-count. Title: 10 words; Abstract: 114 words; Text: 1614 words; References: 8; Figures: 1; supplementary tables: 2 .

Key-words. Frontotemporal dementia; frontotemporal lobar degeneration; TDP-43; Paget's disease of bone; amyotrophic lateral sclerosis; valosin containing protein.

\begin{abstract}
V C P$ mutations are rare causes of autosomal dominant frontotemporal dementias associated with Paget's disease of bone, inclusion body myopathy and amyotrophic lateral sclerosis. We analyzed the VCP gene in a cohort of 199 FTD patients and identified 7 heterozygous mutations in unrelated families, including 3 novel mutations segregating with dementia. This expands the VCP mutation spectrum and suggest that although VCP mutations are rare (3.5\% in this study) the gene should be analyzed even in absence of the full syndromic complex. Reporting genetic variants with convincing arguments for pathogenicity is important considering the large amount of data generated by next generation sequencing and the growing difficulties to interpret rare genetic variants identified in isolated cases.
\end{abstract}

1. Introduction Frontotemporal dementias (FTD) are neurodegenerative dementias displaying a considerable clinical, genetic and pathologic heterogeneity. Between 20 and $40 \%$ of cases are familial, c9orf72 and GRN genes being, by far, the most common genetic causes of the disease. Many other genes, including valosin containing protein $(V C P)$, contribute for a small proportion of familial cases (Watts et al., 2004). So far, more than 45 VCP mutations have been identified (Supplementary table S1). They are 
responsible for a variable association of inclusion body myopathy (IBM), Paget's disease of bone (PDB), ACCEPTED MANUSCRIPT

FTD and amyotrophic lateral sclerosis (ALS), known as IBMPFD or multisystem proteinopathy (MSP)

(Kim et al., 2013; Al-Obeidi et al., 2018). The common neuropathological substrate of $V C P$ mutations is a frontotemporal lobar degeneration (FTLD) with TDP43-immunoreactive inclusions according to the current harmonised classification (Neumann et al., 2007; Mackenzie et al. 2011). In this paper, we have screened a cohort of 199 FTD patients and identified three novel VCP mutations. We present the phenotypes of mutation carriers and discuss the pathogenicity of the mutations in view of their predicted effect on protein structure and the results of the in silico functional analyses.

\section{Methods}

2.1. Mutation screening and molecular analyses. We sequenced the VCP gene on DNA extracted from lymphocytes by Sanger or exome sequencing in 199 unrelated FTD probands. The most frequent FTD, ALS and MSP genes have been analyzed first by Sanger or exome sequencing (MAPT, GRN, TBK1, SQSTM1, hnRNPA1, hnRNPA2B1, TARDBP, CHMP2B, FUS/TLS, PFN1, UBQLN2) or by repeat primed-PCR (c9orf72), and mutation carriers were excluded from the screening. In three families (families 1, 2, 3) carrying novel VCP mutations, DNA of affected relatives was also analyzed, when possible. In family 1, DNAs of a proband's sib (III-2) and an affected relative (II-3) were available for genetic analyses (Figure 1). In family 2 no affected relatives could be sampled for genetic analyses. One affected relative (III-2) was analyzed in family 3 (Figure 1). Written informed consent for clinic-genetic studies was obtained for all FTD probands and relatives. This study was approved by the local ethical committee (AP-HP-Paris).

\section{Results}

3.1. Molecular analyses. We identified 7 heterozygous missense mutations in 7 unrelated probands (7/199, $3.5 \%$ after exclusion of other FTD mutations). Four probands carried already known mutations (p.Arg93Cys, p.Pro137Leu, p.Arg159His, p.Ala439Pro, supplementary table S1), 3 probands (families 1, 2 and 3) carried novel $V C P$ mutations. A c.374G>A mutation (exon 4, NM_007126), leading to a p.Gly125Asp substitution, was identified in the proband III-1 of family 1, as well as in two affected relatives (II-3, III-2, Figure 1A) and an obligate transmitter (II-2). A c.353C > T, p.Pro118Leu mutation (exon 4) was 
identified in proband of family 2. A c.296T>A, p.Val99Asp (exon 3) was identified in the proband (III-1) ACCEPTED MANUSCRIPT

and one affected relative (III-2) in family 3 . The three novel substitutions affected conserved residues across species, were located in the $\mathrm{N}$-terminal domain of the protein and were absent from public databases of controls (EVS, ExAC, 1000Genome, dbSNP). The 3 mutations were predicted to be probably damaging (Wintervar) or deleterious (PolyPhen, Mutation Taster, SIFT) using four in silico prediction softwares (Supplementary table S2). No mutations were found in other MSP, FTD and ALS genes, thus strongly supporting that the p.Val99Asp, p.Pro118Leu and p.Gly125Asp are disease-causing mutations.

3.2. Probands and families description. The proband III-1 of family 1 (Figure 1A), carrying a c.374G>A, p.Gly125Asp mutation, was a French Caucasian subject who insidiously developed, at age 54, apathy and socially inappropriate behaviors. At the age of 57, he manifested familiarity, loss of empathy, anosognosia, grasp reflex and imitation behaviors. The neuropsychological evaluation disclosed impaired attention, planning abilities, working memory and mental flexibility. His score on the MMSE was 27/30, he scored 14/18 on the frontal assessment battery (FAB) and 6/20 on the Wisconsin card sorting test (WCST), with 2 criteria. The total recall score of the free and cued recall test was 47/48. Brain MRI showed bilateral mesial frontal and perisylvian atrophy, with left predominance (Supplementary Figure S1A, 1B); ${ }^{99 \mathrm{~m}} \mathrm{Tc}-\mathrm{ECD}$ SPECT revealed bilateral mesial frontal hypoperfusion. A behavioral FTD was diagnosed. The patient did not develop clinical symptoms of PDB nor IBM, but was not specifically investigated to detect asymptomatic PDB. He died at age 61. A brain post-mortem examination was performed that revealed marked cranial vault thickening evocative of PDB, and confirmed the diagnostic of FTLD. A proband's sib (III-2) was affected by PDB at the age of 50. In his early sixties, he developed delusional ideas and progressive behavioral disorders with apathy, agitation and impulsiveness. MRI showed fronto-temporal atrophy. A diagnosis of probable FTD was made. Proband's affected parent (II-2) and grandparent (I-1) also had PDB; they presented cognitive and behavioral disorders suggestive of FTD and leading to dementia. They died at age 77 and 74, respectively. Two other relatives (II-3, II-4) developed dementia and died at age 66 and 65. 
The proband III-1 of family 2 (Figure 1B), who carried a c.353C >T, p.Pro118Leu mutation, developed ACCEPTED MANUSCRIPT

progressive behavioral disorders at age 58, characterized by apathy, eating disorders (bulimia) and loss of social conducts. Neuropsychological testing revealed executive dysfunctions, impaired set-shifting on the WCST ( 1 criterion), reduced verbal fluencies ( 4 animals and 2 words beginning with the letter "P" in 2 minutes) but spared episodic memory. ${ }^{99 m}$ Tc-ECD SPECT showed marked frontal hypoperfusion, confirming the diagnosis of bvFTD. The patient was lost to follow up. A proband's sib (III-2) developed verbal semantic disorders at age 64, associated with logorrhea, disinhibition, aggressiveness and executive dysfunctions. Brain MRI and ${ }^{99 \mathrm{~m}}$ Tc-ECD SPECT revealed marked, predominantly left, temporal lateral involvement at the age of 69. Serum alkaline phosphatases were normal. He died at age 70. Their affected parent (II-2) had a history of leg fracture and died of dementia associated with muscular weakness at the age of 53. Two sibs of the affected parent (II-3, II-4) also developed early-onset dementia, before the age of 60. There was no personal or familial history of PDB or myopathy, although not specifically investigated.

The proband III-1 of family 3 carried a c.296T>A, p.Val99Asp mutation (Figure 1C). He developed progressive behavioral disorders at the age of 65 (irritability, familiarity, joviality and rituals). At age 70, his score on the MMSE was $24 / 30$, on the MDRS was $92 / 144$ and $7 / 18$ on the FAB. Brain MRI showed predominantly left, frontotemporal atrophy. ${ }^{99 m}$ Tc-ECD SPECT showed marked, mostly left, frontotemporo-parietal hypoperfusion. He had no muscular symptoms or clinical PDB. He later developed delusions, and died at age 74. An affected sib (III-2) had seizures and cognitive disorders at age 66, characterized by executive dysfunction (FAB score: 14/18) and categorial fluency deficit (animals) (12,

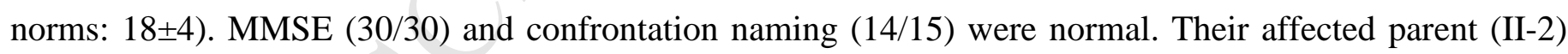
had a history of multiple bone fractures since age 62, and later developed gait disorders (age 63), dementia (age 71) and seizures (age 75). He died at age 78. A parent's sib (II-3) had a bone fracture at age 62, and a PDB was confirmed by bones radiography and elevated serum alkaline phosphatases. He had dementia at age 72 , and died at age 76 .

4. Discussion. We report 3 novel $V C P$ mutations segregating with FTD in 3 unrelated families. Their pathogenicity is highly probable since they affect highly conserved residues, they are predicted to be 
damaging by in silico prediction softwares, and because of the absence of any other MSP and FTD diseasecausing mutations. Furthermore, the variable association of FTD and PDB in patients of families 1 and 3 was highly suggestive of MSP, even in the absence of IBM or ALS. Although absent or not clinically detected in the patients described in this study, IBM is also one of the core features of MSP, present in $90 \%$ of mutation carriers (Al-Obeidi et al., 2018, supplementary Table S1). IBM, FTD, PDB and ALS can be variably associated in the same patient or within families; this inter- and intra-familial clinical variability could be partly due to the effect of genetic (or other) disease phenotype modifiers. $V C P$ is the most frequent gene involved in this syndromic association, followed by SQSTM1 gene (Le Ber et al., 2013). Mutations in the prion-like domains of two other genes coding for heterogeneous nuclear ribonucleoproteins, hnRNPAl and $h n R N P A 2 B 1$, are only rarely involved (Kim et al., 2013). Proband's phenotypes are in line with the well-recognized heterogeneity of $V C P$ mutations, which only rarely manifest with the full symptomatology of MSP. Patients of this study were mainly affected by a behavioral variant of FTD, but the phenotype of patient III-2 in family 1 suggests that verbal semantic deficit can be early associated to behavioral disorders. The p97/VCP protein, encoded by VCP gene, is a member of the AAA-ATPase superfamily including four domains: an N-terminal binding domain, two AAA modules and a C-terminal domain (Watts et al., 2004). It is involved in diverse cellular activities such as transcription, cell cycle control, autophagy, membrane fusion and Golgi reassembly. It also acts as a chaperone, and plays a critical role both in endoplasmic reticulum-associated protein degradation (ERAD), which is fundamental in protein quality control, and in ubiquitin-proteasome degradation system, a major cellular process of protein clearance (Meyer and Wheil, 2014). The p.Val99Asp, p.Pro118Leu and p.Gly125Asp mutations are all located in the N-terminal domain of the protein, where the vast majority of disease-causing mutations have been identified. This domain is responsible for binding to many cofactors, adaptors and ubiquitinated target proteins, similarly to the vast majority of other deleterious $V C P$ mutations. It is thus possible that mutations altering this domain prevent p97/VCP to bind to its natural substrates and to perform its chaperone role in the ubiquitin-proteasome pathway (Tang and Xia, 2016). Functional studies are needed, however, to definitely confirm the pathogenicity and to validate the biological impact of these mutations, as well as identifying additional MSP families carrying these mutations. 
5. Conclusion. This study expands the mutational spectrum of $V C P$, including three novel mutations ACCEPTED MANUSCRIPT

affecting for the first-time codons 99, 118 and 125. Although the relative frequency of mutations is low, this study shows that $V C P$ should be analyzed even in patients with isolated FTD. Finally, reporting genetic variants with convincing arguments for pathogenicity (particularly familial segregation) is important considering the huge amount of data generated by next generation sequencing, and the growing difficulties to interpret rare genetic variants identified in isolated cases.

Acknowledgements. This study was funded by Assistance Publique - Hôpitaux de Paris (Clinical Research and Development Department), PHRC FTLD-exome (promotion AP-HP, to I.L.B.,), the $7^{\text {th }}$ framework programme of the European Union (NEUROMICS, to A.B.) and by "Investissements d'avenir" ANR-10IAIHU-06. All the authors declare that they have no actual or potential conflicts of interest with this work.

\section{References}

Al-Obeidi E, Al-Tahan S, Surampalli A. Genotype-phenotype study in patients with VCP valosin-containing protein mutations associated with multisystem proteinopathy. Clin Genet. 2018;93(1):119-25.

Kim HJ, Kim NC, Wang YD, et al. Mutations in prion-like domains in hnRNPA2B1 and hnRNPA1 cause multisystem proteinopathy and ALS. Nature 2013;495(7442):467-73.

Le Ber I, Camuzat A, Guerreiro R, et al. SQSTM1 mutations in French patients with frontotemporal dementia or frontotemporal dementia with amyotrophic lateral sclerosis. JAMA Neurol 2013 70(11):140310.

Mackenzie, IRA, Neumann, M, Baborie, A, et al. A harmonized classification system for FTLD-TDP pathology. Acta Neuropathol (Berl.) 2011;122:111-3.

Meyer H, Weihl CC. The VCP/p97 system at a glance: connecting cellular function to disease pathogenesis. J Cell Sci 2014;127(Pt 18):3877-83.

Neumann M, Mackenzie IR, Cairns NJ, et al. TDP-43 in the ubiquitin pathology of frontotemporal dementia with VCP gene mutations. J Neuropathol Exp Neurol 2007;66(2):152-7. 
Tang WK, Xia D. Mutations in the Human AAA+ Chaperone p97 and Related Diseases. Front Mol Biosci. 2016;3:79. eCollection 2016.

Watts GD, Wymer J, Kovach MJ, et al. Inclusion body myopathy associated with Paget disease of the bone and frontotemporal dementia is caused by mutant valosin-contaning protein. Nat Genet 2004;36:377-81. 


\section{Figure and legend}

A - Family 1

II

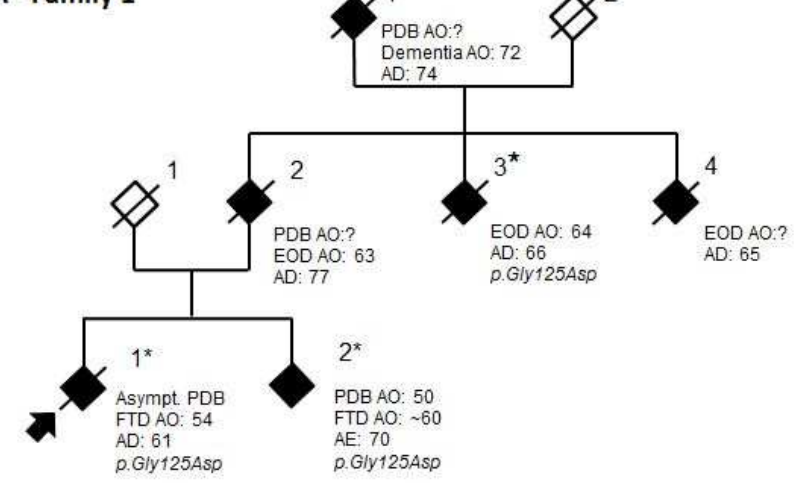

B - Family 2

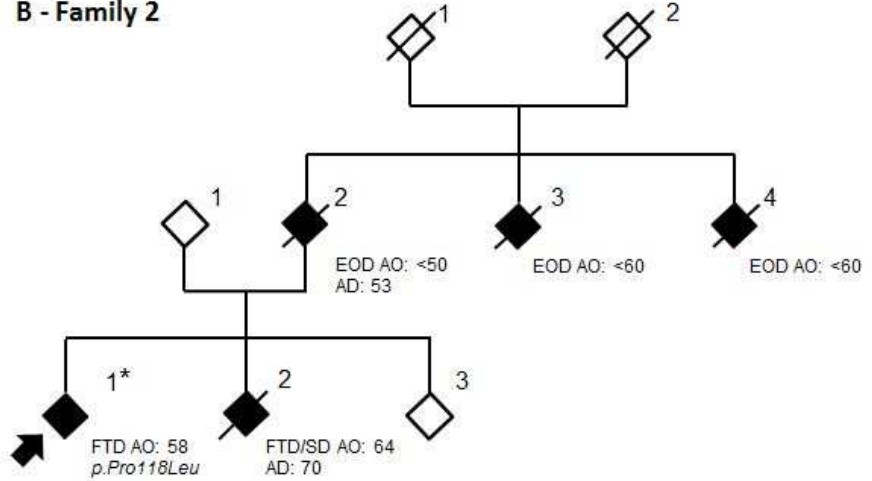

C - Family 3

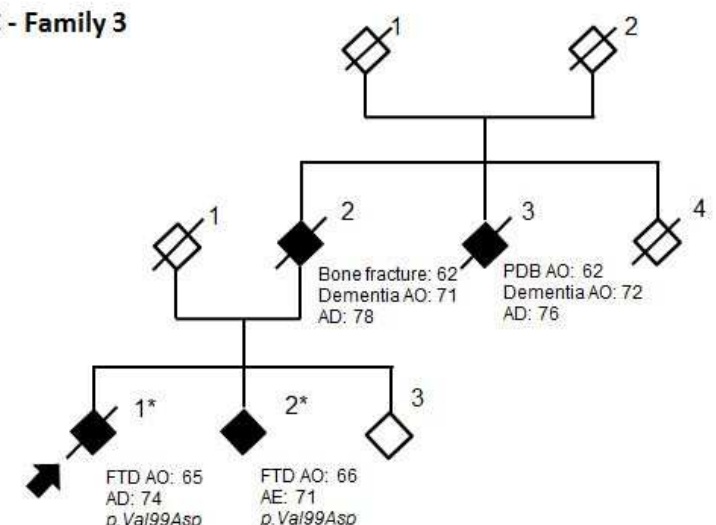

Figure 1. Pedigrees of the families. Individuals affected by FTD and/or PDB are indicated with black diamonds. The probands are indicated by an arrow. Pedigrees have been slightly simplified and gender has been masked to preserve confidentiality. Age at onset (AO), at last examination (AE) or at death (AD) are indicated for each affected individual. Mutation carriers are indicated by a star. A: family 1; II-2 is an obligate transmitter. B: family 2 . C: family 3. Asympt. PBD: asymptomatic Paget disease of bone; EOD: Early onset dementia; FTD: frontotemporal dementia; PDB: Paget's disease of bone; SD: semantic dementia; ?: unknown data. 


\section{Highlights}

Novel $V C P$ mutations expand the mutational spectrum of frontotemporal dementia

1) The frequency of $V C P$ mutations is $3.5 \%$ in FTD.

2) Three novel missense mutations p.Gly125Asp, p.Pro118Leu and p.Val99Asp and their associated phenotypes are reported.

3) VCP mutations should be searched for even in FTD cases without the complete syndrome of multisystem proteinopathy. 


\section{Novel $V C P$ mutations expand the mutational spectrum of frontotemporal dementia}

\section{Verification}

All the authors declare that they have no actual or potential conflicts of interest with this work.

No authors' institutions have contracts relating to this research through which they or any other organization may stand to gain financially now or in the future.

There are no other agreements (of authors or their institutions) that could be seen as involving a financial interest in this work.

This study was funded by Assistance Publique - Hôpitaux de Paris (Clinical Research and Development Department), PHRC FTLD-exome (promotion AP-HP, to I.L.B.,), the $7^{\text {th }}$ framework programme of the European Union (NEUROMICS, to A.B.) and by "Investissements d'avenir" ANR-10-IAIHU-06.

We assure that the data contained in the manuscript being submitted have not been previously published, have not been submitted elsewhere and will not be submitted elsewhere while under consideration at Neurobiology of Aging.

This study was approved by the local ethical committee (AP-HP-Paris).

All authors have reviewed the contents of the manuscript being submitted, approve of its contents and validate the accuracy of the data.

Yours sincerely,

Dario Saracino

Isabelle Le Ber

and all the co-authors 